\title{
Changes in symptom clusters in patients undergoing radiation therapy
}

\author{
Esther Kim • Thierry Jahan • Bradley E. Aouizerat • Marylin J. Dodd • \\ Bruce A. Cooper • Steven M. Paul • Claudia West • Kathryn Lee • Patrick S. Swift • \\ William Wara $\cdot$ Christine Miaskowski
}

Received: 19 December 2008 / Accepted: 2 February 2009 / Published online: 26 February 2009

(C) The Author(s) 2009. This article is published with open access at Springerlink.com

\begin{abstract}
Goals of work The goals of the study were to determine the occurrence rates for and the severity of symptoms at the middle, end, and 1 month after the completion of radiation therapy (RT), to determine the number and types of symptom clusters at these three time points, and to evaluate for changes over time in these symptom clusters.

Materials and methods Symptom occurrence and severity were evaluated using the Memorial Symptom Assessment Scale (MSAS) in a sample of patients $(n=160)$ who underwent RT for breast or prostate cancer. At each time point, an exploratory factor analysis was done to determine the number of symptom clusters (i.e., symptom factors) based on the MSAS symptom severity ratings.

Main results The majority of the patients were male and married with a mean age of 61.1 years. The five symptoms
\end{abstract}

E. Kim • B. E. Aouizerat • M. J. Dodd - B. A. Cooper

S. M. Paul $\cdot$ C. West $\cdot$ K. Lee $\cdot$ C. Miaskowski

School of Nursing, University of California,

San Francisco, CA, USA

T. Jahan $\cdot$ W. Wara

School of Medicine, University of California,

San Francisco, CA, USA

B. E. Aouizerat

Center for Human Genetics, University of California,

San Francisco, CA, USA

P. S. Swift

Alta Bates Comprehensive Cancer Center,

Berkeley, CA, USA

C. Miaskowski $(\triangle)$

Department of Physiological Nursing, University of California,

2 Koret Way, P.O. Box 0610, San Francisco,

CA 94143-0610, USA

e-mail: chris.miaskowski@nursing.ucsf.edu with the highest occurrence rates across all three time points were lack of energy, pain, difficulty sleeping, feeling drowsy, and sweats. Although the number of symptoms and the specific symptoms within each symptom cluster were not identical across the three time points, three relatively similar symptom clusters (i.e., "mood-cognitive" symptom cluster, "sickness-behavior" symptom cluster, "treatmentrelated", or "pain" symptom cluster) were identified in this sample. The internal consistency coefficients for the moodcognitive symptom cluster and sickness-behavior symptom cluster were adequate at $\geq 0.68$.

Conclusions Three relatively stable symptom clusters were found across RT. The majority of the symptom cluster severity scores were significantly higher in patients with breast cancer compared to patients with prostate cancer.

Keywords Symptom clusters · Exploratory factor analysis · Breast cancer - Prostate cancer - Radiation therapy

Sickness behavior

\section{Introduction}

The majority of the research on symptom clusters is crosssectional in nature and has evaluated heterogeneous samples of patients in terms of cancer diagnoses and treatments as well as at different time points in their disease and treatment trajectory (for reviews, see $[2,8,15]$ ). Only a limited number of studies have evaluated how symptom clusters change over the course of chemotherapy or radiation therapy (RT) [1, 5-7, 9-12, 14, 24]. Among these studies, four reported no changes in symptom clusters $[9,10,14,24]$, while six identified differences in the symptom clusters over time $[1,5-7,11,12]$. These inconsistent findings may relate to differences in the 
methods of data collection and/or analyses or to actual differences in patients' symptoms as a result of their disease or treatments.

For example, in these longitudinal studies, the instruments used to measure symptoms and the number of symptoms on each instrument are highly variable. While three studies [7, 9, 24] used comprehensive symptom inventories (i.e., Symptom Checklist of 32 Symptoms, the Edmonton Symptom Assessment System, M. D. Anderson Symptom Inventory) and evaluated symptom severity, the other studies used symptom specific measures or symptom subscales from a quality of life questionnaire $[1,5,6,10-12,14]$.

In addition, several analytic methods were used to determine the symptom clusters. In three studies that evaluated for changes over time in the magnitude of the correlations among symptoms, a number of symptoms were only moderately correlated with other symptoms. However, the strength of these correlations changed over time $[1,5,6]$. In contrast, in studies that used factor analysis to identify changes in symptom clusters $[7,9,10,14]$, the findings are inconsistent.

In a study of lung cancer patients [9], a single cluster of seven symptoms was identified at diagnosis, and this cluster did not change at 3 and 6 months after diagnosis. In another study of breast cancer patients who were receiving chemotherapy or RT [14], two distinct symptom clusters were identified, and these clusters were generally stable across the treatment trajectories. In a study of patients who underwent RT for brain metastasis [7], three symptom clusters were identified at five different time points during RT. Of note, the specific symptoms within each cluster did change over time. Finally, in a longitudinal study of patients with brain tumors who underwent RT [10], the two symptom clusters that were identified at the beginning of RT did not change over the course of RT.

The question of whether symptom clusters are stable or change over time is important both for clinical practice and for the science of symptom cluster research. Given the paucity of research on changes in symptom clusters over time, the purposes of this study, in a sample of patients who underwent RT, were to determine the occurrence and severity of symptoms at the middle, end, and 1 month after the completion of RT, to determine the number and types of symptom clusters at these three time points, and to evaluate for changes over time in these symptom clusters.

\section{Materials and methods}

Participants and settings

This study is part of a descriptive, longitudinal study that evaluated the trajectories of fatigue, pain, and sleep disturbances in oncology outpatients over the course of RT. Patients $(n=160)$ were included if they were adults $(>18$ years of age) who were able to read, write, and understand English, had a Karnofsky Performance Status (KPS) score of $>60$, and were scheduled to receive primary or adjuvant RT. Patients were excluded if they had metastatic disease, had more than one cancer diagnosis, or had a diagnosed sleep disorder. Patients were recruited from RT departments located in a Comprehensive Cancer Center and a community-based oncology program. This study was approved by the Human Subjects Committee at the University of California, San Francisco and at the second study site.

\section{Study procedures}

At the time of the simulation visit (i.e., approximately 1 week prior to the start of RT), patients were approached by a research nurse to discuss participation in the study. After obtaining written informed consent, they were asked to complete a number of baseline questionnaires and symptom inventories. Additional assessments were done over the course of RT and for 4 months after the completion of RT. Demographic and clinical data, as well as data from the Memorial Symptom Assessment Scale (MSAS) [21] that were obtained at the middle, end, and 1 month after the completion of RT, were used in these analyses.

\section{Instruments}

A demographic questionnaire provided information on age, gender, marital status, education, ethnicity, and employment status. Patients completed a checklist of comorbidities and the KPS scale [13]. The KPS is widely used to evaluate the functional status of cancer patients and has well-established validity and reliability [16]. Patients' medical records were reviewed for disease and treatment information.

The MSAS is a self-report questionnaire designed to measure the multidimensional experience of symptoms [21]. The MSAS contains a list of 32 physical and psychological symptoms that occur as a result of cancer or cancer treatment. Using the MSAS, patients were asked to indicate whether or not they had experienced each symptom in the past week (i.e., symptom occurrence). If they had experienced the symptom, they were asked to rate its severity, frequency of occurrence, and distress. Symptom severity was measured using a five-point Likert scale (i.e., $0=$ not at all, $1=$ mild, $2=$ moderate, $3=$ severe, $4=$ very severe). The patients' responses to the severity items were used to create the symptom clusters at the three time points. The reliability and validity of the MSAS is well established [21]. 
Data analysis

All analyses were done using SPSS Version 15 and MPlus version 5.0 [19]. Prior to the symptom cluster analyses, appropriate descriptive statistics were used to generate information on the patients' demographic and clinical characteristics, as well as on symptom occurrence and severity.

At each of the three time points, an exploratory factor analysis (EFA) was performed to determine the number of symptom clusters based on symptom severity ratings. While it is more accurate to describe the results of the EFAs as "symptom factors", in this paper, the term symptom cluster will be used to describe the results of the EFAs. In order to have sufficient variation in the data to perform the EFAs at each time point, symptoms that were present in $\geq 20 \%$ but not more than $80 \%$ of the patients were used in these analyses. While this approach resulted in different numbers of symptoms being included in each EFA, it took into consideration the fact that as patients progress through RT, the occurrence and severity of specific symptoms may change over time.

The major decisions in factor analysis include how to estimate communality, how to determine the number of factors, and how to determine the method for rotating the factors to obtain the simple structure. Polychoric correlations were used to create the matrix of associations because the MSAS items are measured with an ordinal categorical scale [4, 18]. The simple structure was estimated using the method of robust unweighted least squares with geomin (oblique) rotation. The robust unweighted least squares estimator was chosen to achieve more reliable results because of the relatively small sample size (i.e., <200) $[17,18]$.

For each EFA, polychoric correlations were used to create the matrix of associations among 14 symptoms at Time 1 (i.e., middle of RT), among 13 symptoms at Time 2 (i.e., end of RT), and among 12 symptoms at Time 3 (i.e., 1 month after the completion of RT). Factor loadings were considered meaningful if they exceeded 0.30 [4, 20, 26]. The number of factors was considered sufficient to explain the symptom correlations if the model's Chi square was not significant, its comparative fit index (CFI) was $\geq 0.95$, and the root mean square error of approximation (RMSEA) was $\leq 0.06$ [22]. It is possible when using oblique rotation for two items to load on the same factor. If this situation occurred, a particular symptom was included in a factor based on clinical or theoretical plausibility.

While similarities and differences among the symptom clusters found at Times 1, 2, and 3 were examined, no statistical analyses were performed because the best factor structure at each time point was different. For each time point, differences in severity scores (calculated as the mean rating of the items within each symptom cluster) for each of the symptom clusters between patients with breast and prostate cancer were evaluated using the Mann-Whitney two sample rank-sum test. All calculations used actual values. Adjustments were not made for missing data. Therefore, the cohort for each analysis was dependent on the largest set of available data. Differences were considered statistically significant at the $p<0.05$ level.

\section{Results}

Demographic and clinical characteristics

The demographic characteristics of the patients $(n=160)$ at the time of the simulation visit are summarized in Table 1. The majority of the patients were male and married, with a mean age of $61.1(\mathrm{SD}=11.5)$ years. The majority of the patients were Caucasian (72.8\%) and well educated (16.1 \pm 2.9 years of education).

The clinical characteristics of the sample are summarized in Table 2. Over half of the patients had prostate cancer. Almost all of the breast cancer patients (90.9\%) had undergone surgery prior to RT compared to only $9.8 \%$ of the patients with prostate cancer. The mean KPS score for

Table 1 Demographic characteristics of the sample $(n=160)$

\begin{tabular}{ll}
\hline Characteristic & Mean (SD) \\
\hline Age (years) & $61.1(11.5)$ \\
Education (years) & $16.1(2.9)$ \\
& $n(\%)$ \\
Gender & \\
Female & $78(48.7)$ \\
Male & $82(51.3)$ \\
Ethnicity & \\
Caucasian-White & $115(72.8)$ \\
African American & $25(15.8)$ \\
Asian or Pacific islander & $9(5.7)$ \\
Hispanic & $4(2.5)$ \\
Other & $5(3.2)$ \\
Marital status & \\
Married/partnered & $80(51.0)$ \\
Separated or divorced & $33(21.0)$ \\
Never married & $27(17.2)$ \\
Widowed & $9(5.7)$ \\
Not married but living together & $8(5.1)$ \\
Employment status & $69(44.5)$ \\
Employed & $86(55.5)$ \\
Unemployed &
\end{tabular}

$S D$ standard deviation 
Table 2 Clinical characteristics of the sample $(n=160)$

\begin{tabular}{ll}
\hline Characteristic & Mean (SD) \\
\hline Mean number of comorbid conditions & $4.9(2.5)$ \\
Karnofsky Performance Status Score & $92.4(9.7)$ \\
Total dose of radiation administered (cGy) & $6299.1(1020.1)$ \\
& $n(\%)$ \\
Diagnosis & \\
Breast cancer & $78(48.7)$ \\
Prostate cancer & $82(51.3)$ \\
Previous cancer treatment & \\
Surgery & \\
Lumpectomy & $57(74.0)$ \\
Partial mastectomy & $9(11.7)$ \\
Simple mastectomy & $4(5.2)$ \\
Prostatectomy & $8(9.8)$ \\
Chemotherapy & $43(55.8)$ \\
Hormonal therapy & $74(46.3)$ \\
Five most common comorbid conditions & \\
Back problems & $52.8 \%$ \\
Allergies & $47.7 \%$ \\
Arthritis & $35.5 \%$ \\
Hemorrhoids & $34.4 \%$ \\
Headaches & $32.5 \%$ \\
\hline
\end{tabular}

$S D$ standard deviation the sample was $92.4(\mathrm{SD}=9.7)$, the mean number of comorbid conditions was $4.9(\mathrm{SD}=2.5)$, and the types of comorbid conditions were diverse.

Occurrence and severity of symptoms at each time point

The occurrence rates for the symptoms that occurred in $\geq 20 \%$ of the patients at each time point are summarized in Table 3. Across all three time points, the most prevalent symptom was lack of energy, followed by pain, difficulty sleeping, feeling drowsy, and sweats. The mean number of symptoms per patient at the middle, end, and 1 month after the completion of RT was $6.5(\mathrm{SD}=5.3), 6.0(\mathrm{SD}=4.7)$, and $5.9(\mathrm{SD}=5.2)$, respectively. Overall, about $20 \%$ of patients experienced $\geq 10$ symptoms concurrently across all three time points.

The mean severity scores for each symptom at each time point are listed in Table 3. Symptom severity was analyzed by averaging the symptom severity scores for the patients who had the symptom. Lack of energy, difficulty sleeping, sweats, and problem with urination were the four most severe symptoms at all three time points.

Symptom clusters at the middle of RT (Time $1 ; n=152$ )

As shown in Table 4, 14 symptoms were included in EFA at Time 1. A three-factor solution indicated a good fit

Table 3 Occurrence and severity rates for symptoms at each time point

\begin{tabular}{|c|c|c|c|c|c|c|}
\hline \multirow[t]{2}{*}{ Symptoms } & \multicolumn{2}{|c|}{ Middle of RT $(n=152)$} & \multicolumn{2}{|c|}{ End of RT $(n=160)$} & \multicolumn{2}{|c|}{1 month after the completion of RT $(n=132)$} \\
\hline & $\%$ & $\mathrm{M}(\mathrm{SD})$ & $\%$ & $\mathrm{M}(\mathrm{SD})$ & $\%$ & $\mathrm{M}(\mathrm{SD})$ \\
\hline Lack of energy & 55.2 & $1.83(0.66)$ & 59.4 & $1.96(0.73)$ & 51.6 & $1.85(0.69)$ \\
\hline Pain & 50.3 & $1.78(0.75)$ & 51.8 & $1.83(0.76)$ & 39.8 & $1.93(0.64)$ \\
\hline Difficulty sleeping & 47.2 & $1.90(0.83)$ & 47.1 & $1.99(0.81)$ & 47.2 & $1.80(0.85)$ \\
\hline Feeling drowsy & 51.0 & $1.59(0.59)$ & 44.4 & $1.86(0.68)$ & 44.7 & $1.61(0.67)$ \\
\hline Sweats & 42.5 & $2.00(0.75)$ & 39.9 & $1.97(0.75)$ & 36.4 & $1.92(0.81)$ \\
\hline Problems with urination & 35.7 & $1.96(0.59)$ & 37.1 & $1.91(0.76)$ & 35.4 & $1.84(0.74)$ \\
\hline Difficulty concentrating & 38.1 & $1.48(0.64)$ & 35.9 & $1.55(0.69)$ & 31.3 & $1.59(0.72)$ \\
\hline Feeling irritable & 34.7 & $1.54(0.61)$ & 34.0 & $1.67(0.71)$ & 37.1 & $1.62(0.64)$ \\
\hline Itching & 27.0 & $1.63(0.58)$ & 31.9 & $1.90(0.78)$ & 13.6 & $1.59(0.71)$ \\
\hline Worrying & 31.0 & $1.74(0.68)$ & 29.7 & $1.89(0.89)$ & 30.5 & $1.74(0.76)$ \\
\hline Feeling sad & 29.9 & $1.76(0.86)$ & 26.9 & $1.74(0.79)$ & 29.8 & $1.72(0.66)$ \\
\hline Cough & 17.6 & $1.81(0.98)$ & 22.3 & $1.75(0.76)$ & 14.4 & $1.63(0.60)$ \\
\hline Changes in skin & 24.5 & $1.67(0.61)$ & 20.0 & $2.24(0.83)$ & 12.2 & $1.65(0.79)$ \\
\hline Feeling nervous & 20.0 & $1.72(0.65)$ & 18.4 & $1.68(0.67)$ & 20.0 & $1.70(0.64)$ \\
\hline Numbness/tingling in hands/feet & 18.9 & $1.74(0.63)$ & 16.5 & $1.80(0.76)$ & 20.3 & $1.79(0.57)$ \\
\hline Diarrhea & 25.5 & $1.76(0.64)$ & 17.1 & $1.67(0.64)$ & 9.1 & $1.36(0.67)$ \\
\hline
\end{tabular}

Severity scores range from 1 (mild) to 4 (very severe)

$S D$ standard deviation 
Table 4 Factor structure at the middle of radiation therapy $(N=152)$

\begin{tabular}{lccr}
\hline Symptoms & Factor 1 (mood-cognitive symptom) & Factor 2 (sickness-behavior symptom) & Factor 3 (treatment-related symptom) \\
\hline Difficulty concentrating & $\mathbf{0 . 5 5}$ & 0.44 & 0.03 \\
Feeling nervous & $\mathbf{0 . 8 3}$ & 0.03 & 0.08 \\
Feeling sad & $\mathbf{0 . 5 3}$ & 0.48 & -0.01 \\
Worrying & $\mathbf{0 . 6 6}$ & 0.29 & -0.02 \\
Itching & $\mathbf{0 . 5 5}$ & -0.12 & -0.23 \\
Feeling irritable & $\mathbf{0 . 7 0}$ & 0.02 & 0.33 \\
Changes in skin & $\mathbf{0 . 7 3}$ & -0.12 & -0.35 \\
Pain & -0.06 & $\mathbf{0 . 4 7}$ & 0.08 \\
Lack of energy & 0.30 & $\mathbf{0 . 6 5}$ & -0.01 \\
Feeling drowsy & 0.44 & $\mathbf{0 . 4 9}$ & 0.04 \\
Difficulty sleeping & -0.02 & $\mathbf{0 . 5 4}$ & 0.00 \\
Sweats & 0.03 & $\mathbf{0 . 6 0}$ & -0.16 \\
Problem with urination & 0.00 & -0.32 & $\mathbf{1 . 1 6}$ \\
Diarrhea & 0.02 & -0.09 & $\mathbf{0 . 6 2}$ \\
\hline
\end{tabular}

Extraction method: robust unweighted least squares; rotation method: geomin (oblique) rotation

between the data and the model (i.e., $\chi^{2}=29.7, p=0.19$, $\mathrm{CFI}=0.99$, RMSEA=0.04). Seven symptoms were loaded on Factor 1: five psychological symptoms (i.e., difficulty concentrating, feeling sad, feeling nervous, worrying, feeling irritable) and two skin symptoms (i.e., itching, changes in skin). Factor 1 was named the "mood-cognitive" symptom cluster. While itching loaded on the first factor, this symptom was not included in this symptom cluster because it was not related to the other symptoms in the cluster (i.e., correlations between itching and the other symptoms in the cluster ranged from only 0.12 to 0.22 ). However, changes in skin were retained in the cluster because its correlation with other symptoms in the cluster ranged from 0.18 to 0.32 . For the five psychological symptoms within Factor 1, the symptoms were moderately correlated with each other in the range of 0.49 to 0.82 .

Factor 2 contained five symptoms (i.e., pain, lack of energy, feeling drowsy, difficulty sleeping, sweats) and was named the "sickness-behavior" symptom cluster. The third factor contained only two symptoms (i.e., diarrhea, problem with urination) and was named the "treatment-related" symptom cluster.

For the mood-cognitive symptom cluster, the inter-item correlations ranged from 0.18 to 0.82 and the Cronbach's alpha was 0.84 . For the sickness-behavior symptom cluster, the inter-item correlations ranged from 0.14 to 0.63 and the

Table 5 Factor structure at the end of radiation therapy $(N=160)$

\begin{tabular}{lccr}
\hline Symptoms & Factor 1 (mood-cognitive symptom) & Factor 2 (sickness-behavior symptom) & Factor 3 (treatment-related symptom) \\
\hline Difficulty concentrating & $\mathbf{0 . 5 5}$ & 0.34 & 0.06 \\
Feeling sad & $\mathbf{0 . 6 8}$ & 0.32 & -0.01 \\
Worrying & $\mathbf{0 . 9 5}$ & 0.00 & -0.12 \\
Itching & $\mathbf{0 . 3 7}$ & 0.17 & 0.26 \\
Feeling irritable & $\mathbf{0 . 8 3}$ & -0.09 & -0.02 \\
Pain & -0.01 & $\mathbf{0 . 5 8}$ & 0.20 \\
Lack of energy & 0.10 & $\mathbf{0 . 8 4}$ & -0.04 \\
Feeling drowsy & 0.00 & $\mathbf{1 . 1}$ & -0.62 \\
Difficulty sleeping & 0.25 & $\mathbf{0 . 4 4}$ & 0.06 \\
Sweats & 0.17 & $\mathbf{0 . 3 4}$ & 0.18 \\
Problems with urination & 0.13 & -0.01 & $\mathbf{0 . 6 1}$ \\
Changes in skin & 0.48 & 0.00 & $\mathbf{0 . 7 5}$ \\
\hline
\end{tabular}

Extraction method: robust unweighted least squares; rotation method: geomin (oblique) rotation 
Cronbach's alpha was 0.68. For the treatment-related symptom cluster, the inter-item correlation was 0.47 and the Cronbach's alpha was 0.63 .

Symptom clusters at end of RT (Time $2 ; n=160$ )

As shown in Table 5, 12 symptoms were included in the EFA at Time 2. A three-factor solution indicated a good fit between the data and the model (i.e., $\chi^{2}=24.6, p=0.22$, CFI $=0.99$, RMSEA=0.04). The five symptoms in Factor 1 (i.e., difficult concentrating, feeling sad, worrying, itching, feeling irritable) were named the mood-cognitive symptom cluster. The five symptoms in Factor 2 (i.e., pain, lack of energy, feeling drowsy, difficulty sleeping, sweats) were named the sickness-behavior symptom cluster. The two symptoms in Factor 3 (i.e., problem with urination, changes in skin) were named the treatment-related symptom cluster. For the mood-cognitive symptom cluster, the inter-item correlations ranged from 0.23 to 0.65 and the Cronbach's alpha was 0.78 . For the sickness-behavior symptom cluster, the item total correlations ranged from 0.16 to 0.64 and the Cronbach's alpha was 0.73 . For the treatment-related symptom cluster (two items), the inter-item correlation was 0.22 (after reverse coding "problem with urination") and the Cronbach's alpha was 0.36 .

Symptom clusters at 1 month after completion of RT (Time $3 ; n=132$ )

As shown in Table 6, 12 symptoms were used in the EFA at Time 3. A three-factor solution indicated a good fit between the data and the model (i.e., $\chi^{2}=23.3, p=0.22, \mathrm{CFI}=0.98$, RMSEA=0.04). Factor 1 included four symptoms (i.e., difficulty concentrating feeling nervous, feeling sad, worrying) and was named as mood-cognitive symptom cluster. The second factor consisted of five symptoms (i.e., lack of energy, feeling drowsy, difficulty sleeping, problems with urination, feeling irritable) and was named the sickness-behavior symptom cluster. While problem with urination loaded on this factor, this symptom was not included in the symptom factor because it was not related to the other four symptoms. The third factor contained the symptoms of pain, numbness/ tingling in hands/feet, and sweats and was named the "pain" symptom cluster. While sweats loaded on this factor, this symptom was not included in the symptom cluster because it was not conceptually related to the other two symptoms.

For the mood-cognitive symptom cluster, the inter-item correlations ranged from 0.42 to 0.62 and the Cronbach's alpha was 0.81 . For the sickness-behavior symptom cluster, the inter-item correlations ranged from 0.24 to 0.53 and the Cronbach's alpha was 0.70 . For the pain symptom cluster, the inter-item correlation was 0.31 and the Cronbach's alpha was 0.46 .

The similarities and dissimilarities in the symptom clusters across time

The specific symptoms within each symptom cluster at the three time points are summarized in Table 7 . Three distinct symptom clusters were found across the three time points: mood-cognitive symptom cluster, sickness-behavior symptom cluster, and treatment-related or pain symptom cluster. Although the number and specific symptoms within each cluster are not identical, similarities exist in the moodcognitive and sickness-behavior symptom cluster. However, differences over time were noted in the treatment-related or

Table 6 Factor Structure at 1 month after the completion of radiation therapy $(n=132)$

\begin{tabular}{|c|c|c|c|}
\hline Symptoms & Factor 1 (mood-cognitive symptom) & Factor 2 (sickness-behavior symptom) & Factor 3 (pain symptom) \\
\hline Difficulty concentrating & 0.60 & 0.26 & -0.01 \\
\hline Feeling nervous & 0.82 & 0.02 & 0.04 \\
\hline Feeling sad & 0.90 & -0.08 & -0.01 \\
\hline Worrying & 0.81 & 0.13 & -0.04 \\
\hline Lack of energy & -0.01 & 0.71 & 0.27 \\
\hline Feeling drowsy & 0.01 & 0.83 & -0.00 \\
\hline Difficulty sleeping & 0.10 & 0.37 & 0.12 \\
\hline Problems with urination & -0.49 & 0.42 & 0.07 \\
\hline Feeling irritable & 0.28 & 0.51 & 0.06 \\
\hline Pain & -0.11 & 0.16 & 0.49 \\
\hline Numbness/tingling in hands/feet & 0.00 & -0.01 & 1.02 \\
\hline Sweats & 0.06 & 0.31 & 0.42 \\
\hline
\end{tabular}

Extraction method: robust unweighted least squares; rotation method: geomin (oblique) rotation 
Table 7 Symptom clusters and reliability estimates across time points

\begin{tabular}{llll}
\hline & Middle of RT & End of RT & 1month after the completion of RT \\
\hline Mood-cognitive symptom cluster & Difficulty concentrating & Difficulty concentrating & Difficulty concentrating \\
& Feeling sad & Feeling sad & Weeling sad \\
& Worrying & Worrying & Forrying \\
& Feeling irritable & Feeling irritable & Feeling nervous \\
& Feeling nervous & & Itching \\
& Changes in skin & & 0.81 \\
Cronbach's alpha & 0.84 & 0.78 & Lack of energy \\
Sickness-behavior symptom cluster & Pain & Pain & Feeling drowsy \\
& Lack of energy & Lack of energy & Difficulty sleeping \\
& Feeling drowsy & Feeling drowsy & Feeling irritable \\
Cronbach's alpha & Difficulty sleeping & Difficulty sleeping & 0.70 \\
Treatment-related or pain symptom cluster & Sweats & Sweats & Pain \\
Cronbach's alpha & 0.68 & 0.73 & Numbness/tingling in hands/feet
\end{tabular}

pain symptom clusters. Specifically, at the middle of RT, problem with urination and diarrhea clustered together, while problem with urination and skin problems associated with treatment clustered together at the end of RT. Furthermore, a new symptom cluster of pain emerged at 1 month after completion of RT.

To determine the validity of the symptom clusters, differences in symptom severity scores, at each time point, between patients with breast and prostate cancer were evaluated using the Mann-Whitney $U$ test. As shown in Table 8 , at every time point and for every symptom cluster except the treatment-related symptom cluster at the middle of RT (i.e., problem with urination, diarrhea), the patients with breast cancer reported significantly higher symptom cluster severity scores than the patients with prostate cancer.

\section{Discussion}

To our knowledge, this study is the first to describe the occurrence rates and severity scores for the most common symptoms experienced by patients over the course of RT as well as changes over time in symptom clusters in these patients. Of note, lack of energy, pain, and difficulty sleeping were the most prevalent symptoms reported by patients at the middle, the end, and 1 month after the completion of RT. This finding is consistent with previous

Table 8 Differences in the mean symptom severity scores for the three symptom clusters between patients with breast and prostate cancer

\begin{tabular}{|c|c|c|c|c|c|c|c|}
\hline \multirow[t]{2}{*}{ Symptom cluster } & \multirow[t]{2}{*}{ Patient group } & \multicolumn{2}{|c|}{ Middle of RT } & \multicolumn{2}{|l|}{ End of RT } & \multicolumn{2}{|c|}{1 month after the completion of RT } \\
\hline & & $M(\mathrm{SD})$ & $p$ value $^{\mathrm{a}}$ & $\mathrm{i}(\mathrm{SD})$ & $p$ value $^{\mathrm{a}}$ & $M(\mathrm{SD})$ & $p$ value $^{\mathrm{a}}$ \\
\hline Mood-cognitive & $\begin{array}{l}\text { Breast cancer } \\
\text { Prostate cancer }\end{array}$ & $\begin{array}{l}0.68(0.61) \\
0.28(0.51)\end{array}$ & 0.000 & $\begin{array}{l}0.67(0.61) \\
0.31(0.49)\end{array}$ & 0.000 & $\begin{array}{l}0.67(0.76) \\
0.22(0.40)\end{array}$ & 0.000 \\
\hline Sickness-behavior & $\begin{array}{l}\text { Breast cancer } \\
\text { Prostate cancer }\end{array}$ & $\begin{array}{l}1.00(0.64) \\
0.66(0.60)\end{array}$ & 0.002 & $\begin{array}{l}1.02(0.67) \\
0.53(0.55)\end{array}$ & 0.000 & $\begin{array}{l}0.87(0.68) \\
0.53(0.55)\end{array}$ & 0.007 \\
\hline Treatment-related or pain & $\begin{array}{l}\text { Breast cancer } \\
\text { Prostate cancer }\end{array}$ & $\begin{array}{l}0.12(0.35) \\
0.97(0.83)\end{array}$ & 0.000 & $\begin{array}{l}2.40(0.55) \\
1.39(0.48)\end{array}$ & 0.000 & $\begin{array}{l}0.87(0.69) \\
0.38(0.53)\end{array}$ & 0.02 \\
\hline
\end{tabular}

$M$ mean symptom severity score, SD standard deviation, $R T$ radiation therapy

${ }^{a}$ Mann-Whitney $U$ test 
reports of fatigue in patients who underwent RT and chemotherapy [3, 7, 23]. In addition, lack of energy, difficulty sleeping, sweats, and problem with urination were the most severe symptoms at all time points. However, while the occurrence rate for pain decreased over time, the severity of pain appeared to increase over time. Additional research is needed to determine the specific etiologies for the pain.

Another interesting finding is the relatively high occurrence rate for sweats, as well as its relatively high severity rating compared to other symptoms. Previous studies that used the MSAS reported that sweats occurred in 33.6\% [25] to $40.0 \%$ [21] of patients with a severity rating of 1.67 $(\mathrm{SD}=0.83)$ [25]. The relatively high occurrence rates and severity scores for sweats in this sample may be associated with biologically or chemically induced menopause in both the patients with breast and prostate cancer. In fact, across the three measurement times, $24.3 \%$ to $32.1 \%$ of the men and $55.2 \%$ to $58.0 \%$ of women in this study reported sweats. This finding warrants additional investigation.

Although the numbers as well as the specific symptoms within each symptom cluster were not identical across the three time points, three relatively similar symptom clusters (i.e., mood-cognitive symptom cluster, sickness-behavior symptom cluster, treatment-related or pain symptom cluster) were found over time. Of note, both the mood-cognitive symptom cluster $[7,10]$ and the sickness-behavior symptom cluster $[7,24]$ were found in other studies of patients who underwent RT. For example, in a longitudinal study of patients with brain metastasis who underwent to RT [7], the symptoms of anxiety and depression clustered together over the course of RT (i.e., five measures). In addition, in a study of patients with brain tumors [10], the symptoms of feeling nervous, feeling sad, and depressed mood formed a mood cluster at both the beginning and the end of RT.

The symptoms within the sickness-behavior symptom cluster found in this study were relatively stable over time. In fact, lack of energy, feeling drowsy, and difficulty sleeping were present at all three time points. The stability of these three symptoms is consistent with two previous longitudinal RT studies [7, 24] and suggests that these symptoms require systematic assessment and management in patients who undergo RT. Of note, the internal consistency coefficients for this symptom cluster were consistently high across the three time points (i.e., Cronbach's alphas ranged from 0.68 to 0.70 ) in this study as well as in the study by Chow and colleagues [7] (Cronbach's alphas ranged from 0.65 to 0.77 ).

A unique finding in this study is the treatment-related or pain symptom cluster that changed over time. At the middle of RT, the treatment-related symptom cluster consisted of the symptoms of diarrhea and problem with urination. Further examination of the data demonstrated that both of these symptoms occurred with a higher frequency in the patients with prostate cancer compared to those with breast cancer (i.e., problem with urination $=65.3 \%$ versus $5.6 \%$ $\chi^{2}=55.43, p<0.0001$; diarrhea $=40.5 \%$ versus $8.6 \% ; \chi^{2}=$ 19.92, $p<0.0001)$. At the end of RT, the treatment-related symptom cluster consisted of problem with urination and changes in skin. At this time point, $70 \%$ of the men reported a problem with urination compared to only $4.3 \%$ of women $\left(\chi^{2}=64.74, p<0.0001\right)$. In contrast, $43.1 \%$ of the women reported changes in skin compared to only $4.0 \%$ $\left(\chi^{2}=29.24, p<0.0001\right)$ of the men, respectively. Finally, 1 month after the completion of RT, a new symptom cluster of pain and numbness/tingling in hands/feet was identified. All of these symptoms occurred more frequently in the women with breast cancer than in the men with prostate cancer (i.e., pain $=50 \%$ versus $32.4 \% ; \chi^{2}=4.02, p=0.045$; numbness/tingling $=32.7 \%$ versus $11.0 \% ; \chi^{2}=9.183, p=$ 0.002). Taken together, these findings suggest that future studies of symptom clusters in patients undergoing RT may need to be done within cancer diagnoses to better determine treatment-related symptom clusters.

Several limitations of this study need to be mentioned. The sample size was relatively small and did not allow for separate evaluations of symptom clusters in patients with breast and prostate cancer. In addition, as with other longitudinal studies, dropouts occurred over time. Therefore, the sample size for each analysis was different. In addition, because the prevalence rates for the various symptoms changed overtime, different symptoms were entered into the various EFAs. Some of these limitations may be overcome with large samples of patients.

Despite these limitations, the findings from this study suggest that a mood-cognitive and a sickness-behavior symptom clusters occur in patients during the course of RT. However, the specific symptoms within two symptom clusters were not consistent over time. This finding suggests that the need for the symptom cluster to remain stable over time may not be an essential element of the definition of a symptom cluster. This finding warrants replication in future longitudinal studies. In addition, treatment-related symptom clusters that appear to be diagnosis specific warrant additional investigation.

Acknowledgments Drs. Miaskowski and Dodd's research programs are supported by grants from the National Cancer Institute and the National Institute of Nursing Research (NINR). Dr. Aouizerat is funded through the National Institutes of Health Roadmap for Medical Research Grant (8 K12 RR023262). Dr. Kim was funded during her doctoral program through a T32 Grant (NR07088) from the NINR.

Open Access This article is distributed under the terms of the Creative Commons Attribution Noncommercial License which permits any noncommercial use, distribution, and reproduction in any medium, provided the original author(s) and source are credited. 


\section{References}

1. Ahlberg K, Ekman T, Gaston-Johansson F (2005) Fatigue, psychological distress, coping resources, and functional status during radiotherapy for uterine cancer. Oncol Nurs Forum 32:633-640. doi:10.1188/05.ONF.633-640

2. Barsevick AM (2007) The concept of symptom cluster. Semin Oncol Nurs 23:89-98. doi:10.1016/j.soncn.2007.01.009

3. Bender CM, Ergyn FS, Rosenzweig MQ, Cohen SM, Sereika SM (2005) Symptom clusters in breast cancer across 3 phases of the disease. Cancer Nurs 28:219-225. doi:10.1097/00002820200505000-00011

4. Brown TA (2006) Confirmatory factor analysis for applied research. Guilford, New York

5. Byar KL, Berger AM, Bakken SL, Cetak MA (2006) Impact of adjuvant breast cancer chemotherapy on fatigue, other symptoms, and quality of life. Oncol Nurs Forum 33:E18-E26. doi:10.1188/ 06.ONF.E18-E26

6. Chan CW, Richardson A, Richardson J (2005) A study to assess the existence of the symptom cluster of breathlessness, fatigue and anxiety in patients with advanced lung cancer. Eur J Oncol Nurs 9:325-333. doi:10.1016/j.ejon.2005.02.003

7. Chow E, Fan G, Hadi S, Wong J, Kirou-Mauro A, Filipczak L (2008) Symptom clusters in cancer patients with brain metastases. Clin Oncol (R Coll Radiol) 20:76-82. doi:10.1016/j.clon.2007.09.007

8. Fan G, Filipczak L, Chow E (2007) Symptom clusters in cancer patients: a review of the literature. Curr Oncol 14:173-179. doi: $10.3747 /$ co. 2007.145

9. Gift AG, Stommel M, Jablonski A, Given W (2003) A cluster of symptoms over time in patients with lung cancer. Nurs Res 52:393-400. doi:10.1097/00006199-200311000-00007

10. Gleason JF Jr, Case D, Rapp SR, Ip E, Naughton M, Butler JM Jr, McMullen K, Stieber V, Saconn P, Shaw EG (2007) Symptom clusters in patients with newly-diagnosed brain tumors. J Support Oncol 5(427-433):436

11. Hadi S, Fan G, Hird AE, Kirou-Mauro A, Filipczak LA, Chow E (2008) Symptom clusters in patients with cancer with metastatic bone pain. J Palliat Med 11:591-600. doi:10.1089/jpm.2007.0145

12. Hadi S, Zhang L, Hird A, de Sa E, Chow E (2008) Validation of symptom clusters in patients with metastatic bone pain. Curr Oncol 15:211-218

13. Karnofsky D, Abelmann WH, Craver LV, Burchenal JH (1948) The use of nitrogen mustard in the palliative treatment of cancer. Cancer 1:634-656. doi:10.1002/1097-0142(194811)1:4<634:: AID-CNCR2820010410>3.0.CO;2-L
14. Kim HJ, Barsevick AM, Tulman L, McDermott PA (2008) Treatment-related symptom clusters in breast cancer: a secondary analysis. J Pain Symptom Manage 36:468-479. doi:10.1016/j. jpainsymman.2007.11.011

15. Miaskowski C, Aouizerat BE (2007) Is there a biological basis for the clustering of symptoms? Semin Oncol Nurs 23:99-105. doi:10.1016/j.soncn.2007.01.008

16. Mor V, Laliberte L, Morris JN, Wiemann M (1984) The Karnofsky Performance Status Scale. An examination of its reliability and validity in a research setting. Cancer 53:2002 2007. doi:10.1002/1097-0142(19840501)53:9<2002::AIDCNCR2820530933>3.0.CO;2-W

17. Muthen BO, Kaplan D (1985) A comparison of some methodologies for the factor analysis of non-normal Likert variables. Br J Math Stat Psychol 38:171-189

18. Muthen LK, Muthen BO (1998-2008) Mplus User's Guide (5th ed.). Muthen \& Muthen, Los Angeles

19. Muthen LK, Muthen BO (2008) Mplus (Version 5.1). Muthen \& Muthen, Los Angles

20. Pett MA, Lackey NR, Sullivan JJ (2003) Making sense of factor analysis: the use of factor analyis for instrument development in health care research. Sage, Thousand Oaks, CA

21. Portenoy RK, Thaler HT, Kornblith AB, Lepore JM, Friedlander-Klar H, Kiyasu E, Sobel K, Coyle N, Kemeny N, Norton L et al (1994) The Memorial Symptom Assessment Scale: an instrument for the evaluation of symptom prevalence, characteristics and distress. Eur J Cancer 30A:1326-1336. doi:10.1016/0959-8049(94) 90182-1

22. Raykov T, Marcoulides GA (2006) A first course in structural equation modeling. Lawrence Erlbaum, Mahwah

23. Wang SY, Tsai CM, Chen BC, Lin CH, Lin CC (2008) Symptom clusters and relationships to symptom interference with daily life in Taiwanese lung cancer patients. J Pain Symptom Manage 35:258-266. doi:10.1016/j.jpainsymman.2007.03.017

24. Wang XS, Fairclough DL, Liao Z, Komaki R, Chang JY, Mobley GM, Cleeland CS (2006) Longitudinal study of the relationship between chemoradiation therapy for non-small-cell lung cancer and patient symptoms. J Clin Oncol 24:4485-4491. doi:10.1200/ JCO.2006.07.1126

25. Yan H, Sellick K (2004) Symptoms, psychological distress, social support, and quality of life of Chinese patients newly diagnosed with gastrointestinal cancer. Cancer Nurs 27:389-399. doi:10.1097/00002820-200409000-00009

26. Zwick WR, Velicer WF (1982) Factors influencing four rules for determining the number of components to retain. Multivariate Behav Res 17:253-269. doi:10.1207/s15327906mbr1702 5 